\title{
Selective launching of higher-order modes into an optical fiber with an optical phase shifter
}

\author{
W. Q. Thornburg, B. J. Corrado, and X. D. Zhu \\ Department of Physics, University of California, Davis, Davis, California 95616
}

Received September 13, 1993

\begin{abstract}
We demonstrate a simple, effective technique for launching higher-order modes into a double-mode optical fiber by using an optical phase shifter at the input end. The suppression of the lowest-order mode was measured (but not limited) to be $27 \mathrm{~dB}$.
\end{abstract}

There has long been interest in and effort for efficient and selective launching and detection of loworder modes into a low-mode-number optical fiber. ${ }^{1-6}$ Researchers have been motivated in part by the potential application of a single fiber to carry more than one information channel. A number of techniques have been developed and demonstrated to achieve such a goal. ${ }^{1-5}$ For example, Youngquist et $a .^{2}{ }^{2}$ showed that by periodically stressing a doublemode fiber the phase-velocity mismatch between the first-order and the second-order modes can be effectively compensated for and the power of the former can then be coupled selectively to the latter. The same group later showed another highly selective method for launching a second-order mode by using an evanescent directional coupler. ${ }^{1}$ In this case, the phase velocity of the lone guided mode in a single-mode fiber is matched to that of a secondorder mode in a double-mode fiber. They were able to achieve a $90 \%$ coupling efficiency and a suppression of the launched power to first-order modes by at least $24 \mathrm{~dB}$. In these methods, the phase velocities of all modes in the fibers must be measured precisely so that high coupling efficiency and selectivity can be ensured. To avoid the backward power coupling, one must select the interaction distances properly as well.

In this Letter we demonstrate that a simple setup involving an optical phase shifter is capable of selectively launching a higher-order mode into a lowmode-number polarization-preserving fiber. The ratio of the launched power into an $L P_{11 \text {,even }}$ mode to that into an $\mathrm{LP}_{01}$ mode is greater than $18 \mathrm{~dB}$. The suppression of the latter power alone was measured to be at least $27 \mathrm{~dB}$. The simplicity of this method in the mode launch and separation processes is desirable for using low-mode-number fiber to carry multiple information channels in a single fiber.

In contrast to previous methods, which exploit the longitudinal phase of low-order modes, the present technique takes advantage of the phase variations over a fiber cross section for various low-order modes. ${ }^{1-5}$ Consider a weakly guided double-mode fiber. The phase of a first-order mode does not vary over the fiber cross section, whereas the phase distribution of a second-order mode is usually symmetric in magnitude but changes sign about one of the symmetry axes of the fiber. To launch the first-order mode with an effective suppression of higher-order modes, one starts with a collimated beam with a symmetric phase and amplitude front. To launch a second-order mode with the same selectivity is not so straightforward without launching power into the first-order mode. However, if the phase front of the collimated beam is changed to match that of a selected higher-order mode, the latter should be selectively launched just as well by symmetry consideration. This is what is described in this Letter. The phase change is achieved by a phase shifter made of two dielectric parallel plates with straight edges. The edges adjoin along a symmetry axis of a fiber. By tilting one of the plates, we change the optical path length for a laser beam passing through the plate. In particular, we can make one half of the phase front of an incident beam to be $180^{\circ}$ out of phase from the other half before the beam is launched into the fiber.

Specifically, consider a cylindrically symmetric step-index optical fiber. The spatial distribution of the electric field of a guided mode is described mainly by Bessel functions modulated by azimuthal cosine and sine functions $J_{\nu}(u R)\left[\begin{array}{c}\cos (\nu \Phi) \\ \sin (\nu \Phi)\end{array}\right]$ in the core and $K_{\nu}(w R)\left[{ }_{\sin (\nu \Phi)}^{\cos (\nu \Phi)}\right]$ in the cladding. ${ }^{7}$ Here $R=\rho / a$, where $\rho$ is the radial distance from the center of the core and $a$ is the core radius, $u$ and $w$ are the dimensionless parameters determined by the fiber characteristics and the optical frequency, and $\nu$ is the order of the mode. In the weakly guiding limit, where $\Delta=1 / 2\left(1-n_{\text {cladding }}^{2} / n_{\text {core }}^{2}\right) \ll 1$, the linear polarization approximation can be applied. ${ }^{7}$ The electricfield distributions of the first six guided modes are shown in Fig. 1. The arrows indicate the direction of the electric fields. There are two first-order modes $\left(\mathrm{LP}_{01}\right)$ : one polarized along the $y$ axis, and the other polarized along the $x$ axis. They are both described by $J_{0}(u R)$ with a constant phase front. There are four second-order modes $\left(L P_{11}\right)$, which are made of linear combinations of $\mathrm{TE}_{01}$ and $\mathrm{TM}_{01}$ modes with $\mathrm{HE}_{21 \text {,even }}$ and $\mathrm{HE}_{21 \text {,odd }}$ modes, respectively. They are described by $J_{1}(u R)\left[\begin{array}{l}\cos (\Phi) \\ \sin (\Phi)\end{array}\right]$, which change signs about one of the symmetry axes ( $x$ or $y$ axis) of the fiber. ${ }^{7}$ All six modes may be launched selectively by choice 


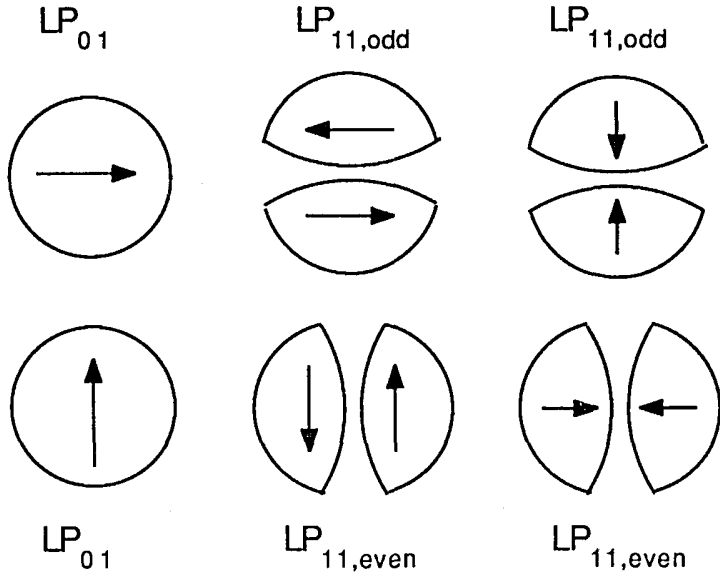

Fig. 1. Electric-field distributions of the first six modes in a weakly guided, cylindrically symmetric fiber. The arrows indicate the directions of the fields.

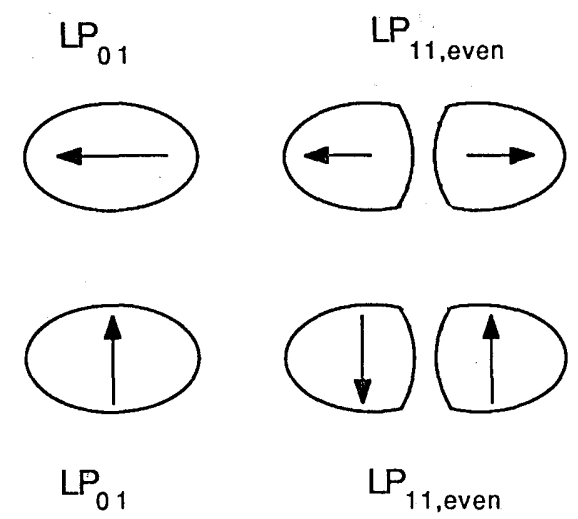

Fig. 2. Electric-field distributions of the first four guided modes in an elliptical-core fiber. The arrows indicate the primary directions of the electric fields. The nodal lines for $\mathrm{LP}_{11 \text {,even }}$ modes are along the short axis ( $y$ axis) of the ellipse.

of the proper polarization and phase front of the incident beam.

We demonstrate this principle with a polarizationpreserving elliptical-core fiber donated by Andrew Corporation. ${ }^{8}$ The choice is to avoid the effect of strain-induced birefringence. ${ }^{8-12}$ The fiber is designed for single-mode transmission at $830 \mathrm{~nm}$ with a numerical aperture $\left(n_{\text {core }}^{2}-n_{\text {cladding }}^{2}\right)^{1 / 2}=0.3$. The core is a $1.3 \mu \mathrm{m} \times 2.6 \mu \mathrm{m}$ ellipse. A $2-\mathrm{mW} \mathrm{He}-\mathrm{Ne}$ laser operated at $632.8 \mathrm{~nm}$ in a $\mathrm{TEM}_{00}$ mode is used as the source. The fiber supports two $\mathrm{LP}_{01}$ modes and two $\mathrm{LP}_{11 \text {,even }}$ modes with nodal lines along the short axis ( $y$ axis) of the ellipse, as shown in Fig. $2^{8-12}$ The propagation constants for the two $\mathrm{LP}_{01}$ modes (and for the two $\mathrm{LP}_{11}$ modes) are separated by approximately $3-4 \mathrm{rad} / \mathrm{mm}$, so that all four modes are nondegenerate. ${ }^{10}$ In our experiment we launch the first-order $\mathrm{LP}_{01}$ and the second-order $\mathrm{LP}_{11 \text {,even }}$ modes that are polarized along the $y$ axis.

The setup is shown in Fig. 3. The fiber is held with a Newport F-925 fiber coupler. The phase shifter is made of two identical rectangular microscope slides $2 \mathrm{~mm}$ thick. The slides have parallelness of $5 \times 10^{-5} \mathrm{rad}$, so that the wave-front distortion through each slide has negligible effects on the selective launching process. The straight edges of the two slides adjoin along the short axis ( $y$ axis) of the fiber with a small separation of $0.12 \mathrm{~mm}$. The separation facilitates the rotation of one of the slides about the $x$ axis. The incident $\mathrm{He}-\mathrm{Ne}$ beam has a diameter of $1.5 \mathrm{~mm}$ and is polarized along the $y$ axis. It is focused down with a $20 \times$ microscope objective that has a focal length of $8.9 \mathrm{~mm}$. The beam waist size at the focal plane is estimated to be $3 \mu \mathrm{m}$, and it has an area approximately 3 to 4 times the size of the elliptical core. This makes the alignment a simple task, given the coarse adjustment on the F-925 fiber coupler. The fiber is placed near the foca plane of the beam. We optimize the longitudinal position by maximizing the launched power. To launch the $\mathrm{LP}_{01}$ mode we pass the $\mathrm{He}-\mathrm{Ne}$ beam normally through one of the two glass slides on the phase sifter. The maximum launched power into the $\mathrm{LP}_{01}$ was measured to be $38 \%$ of the incident power. This is consistent with the areal ratio of the incident beam to the elliptical core. The output beam from the fiber is recollimated with the second microscope objective. The resultant intensity distribution along the long axis of the fiber is depicted in Fig. 4. The solid curve is a fit to a Gaussian function. ${ }^{13}$

To launch the second-order mode $\mathrm{LP}_{11 \text {,even }}$ with the minimum power into the $\mathrm{LP}_{01}$ mode, we pass the

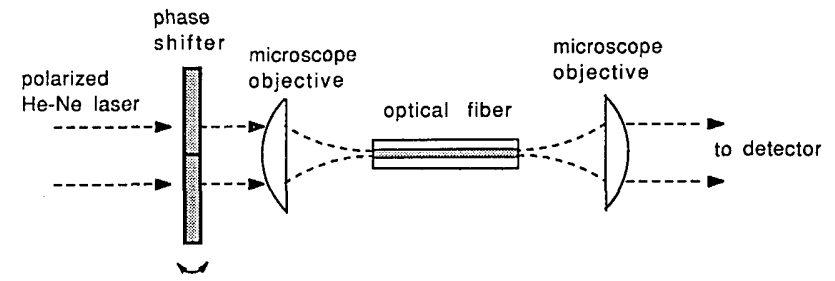

Fig. 3. Simplified experimental setup. The phase shifter is made of two rectangular microscope glass slides, whose adjoining edges are along the short axis ( $y$ axis) of the elliptical fiber. One of the slides is rotatable about the $x$ axis so that the optical path can be changed.

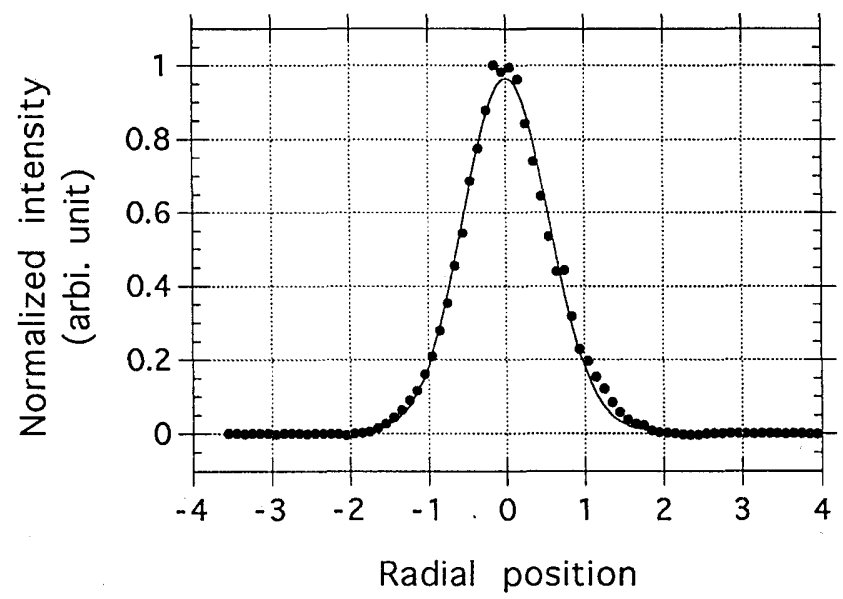

Fig. 4. Measured intensity distribution after the second microscope objective when the incident beam passes through one glass slide only. The solid curve is a fit to a Gaussian function. The absolute transmittance, after reflection correction, is $38 \%$. 


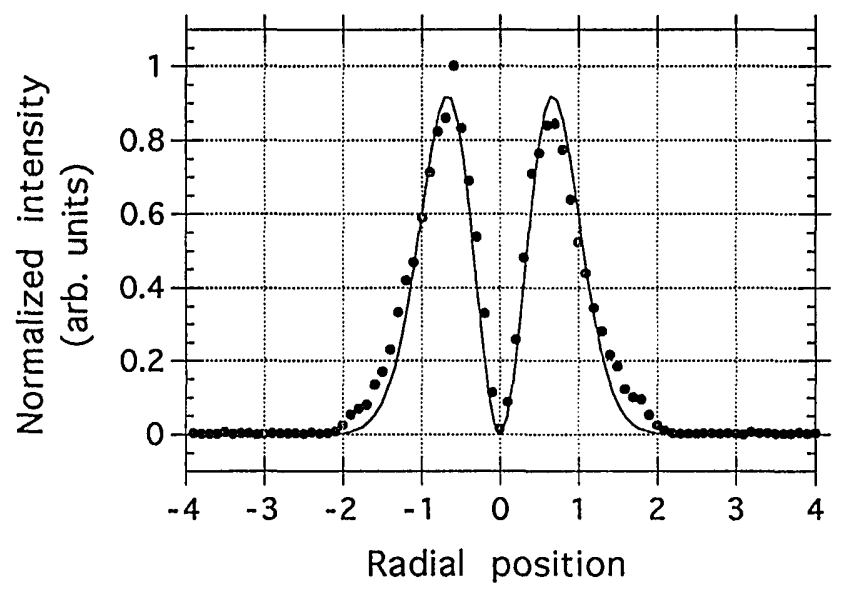

Fig. 5. Measured intensity distribution after the second microscope objected when the incident beam passes through both slides with the adjoining edges bisecting the beam cross section. The solid curve is a fit to the product of a Gaussian function and the first-order Hermite polynomial. The absolute transmittance, after reflection correction, is $8.4 \%$.

$\mathrm{He}-\mathrm{Ne}$ beam normally through both glass slides on the phase shifter with the adjoining edges bisecting the beam cross section. By rotating one of the slides about the $x$ axis, we change the phase front on one half of the beam cross section by $180^{\circ}$ from that on the other half to match the phase distribution of the $\mathrm{LP}_{11, \text { even }}$ mode. We observed that the output intensity distribution after the second objective lens changed from that corresponding to $\mathrm{LP}_{01}$ to that corresponding to $L P_{11, \text { even }}$ as shown in Fig. 5. The solid curve is a fit to the product of the first-order Hermite polynomial and a Gaussian function. ${ }^{13}$ The width of the Gaussian function is $10 \%$ smaller than that for the first-order mode shown in Fig. 4. The tilting was optimized by minimization of the power near the nodal line. The total launched power into the $\mathrm{LP}_{11 \text {,even }}$ mode is $22 \%$ of that into the $\mathrm{LP}_{01}$ mode. $\mathrm{A}$ theoretical upper limit is roughly $25 \%$, which agrees well with the experimental observation. ${ }^{13}$ The intensity near the nodal line is $2 \%$ of that at the peak of the $L P_{11 \text { even }}$ mode. This gives a suppression factor of the $\mathrm{LP}_{01}$ power of at least 460 , or $27 \mathrm{~dB}$. The relative suppression ratio of the launched $\mathrm{LP}_{01}$ power to the launched $\mathrm{LP}_{11 \text {,even }}$ power is $18 \mathrm{~dB}$, or a factor of 63 . These ratios are currently limited by the $0.12-\mathrm{mm}$ separation between the two glass slides; therefore they should be improved linearly with a decrease in the separation. To make sure that the wave-front distortion through the glass slides indeed has a negligible effect on the mode launching, we increased the phase difference by tilting the rotating glass slide further, and we could completely restore the $\mathrm{LP}_{01}$ mode as indicated by the output intensity distribution.

We now discuss the results. We first note that the launching efficiency, which is low in the present case, can easily be improved with microscope objective lenses of higher magnification. Furthermore, this technique is easily applied to the launching of other higher-order modes with the same selectivity. In fact, one can even add a second phase shifter with the nodal edge orthogonal to the first one. This enables one to launch even higher-order guided modes. An important feature of this method is its simplicity. One can use the method in the receiving end of a fiber to separate different modes. For example, an $\mathrm{LP}_{01}$ mode and an $\mathrm{LP}_{11 \text {,even }}$ mode in a double-mode fiber may be separately analyzed at the exit by splitting the output beam into two parts. With one part launched into an analyzing single-mode fiber without a phase shifter, the output come only from the $\mathrm{LP}_{01}$ mode. With the other part launched into a separate single-mode fiber through a phase shifter, the output comes only from the $L P_{11 \text {,even }}$ mode. If each mode carries a distinct information channel, we have two channels in a single fiber. In practice, a phase shifter can be made through high-precision coating processes in an integrated all-optical system. Finally, it is vital that one use polarization-preserving fibers to minimize mode mixing so that more than one mode can be used over a long distance suitable for communication. ${ }^{8-12}$

In conclusion, we have demonstrated a simple technique to launch and analyze higher-order modes into a low-mode-number fiber with high selectivity. With polarization-preserving fibers and improvements in the present setup, it should be possible and practical to use low-mode-number multimode fiber to carry more information channels.

We are indebted to K. Toyama, H. J. Shaw, M. J. F. Digonnet, and R. Dyott for helpful discussions.

\section{References}

1. W. V. Sorin, B. Y. Kim, and H. J. Shaw, Opt. Lett. 11, 581 (1986).

2. R. C. Youngquist, J. L. Brooks, and H. J. Shaw, Opt. Lett. 9, 177 (1984).

3. C. D. Poole, C. D. Townsend, and K. T. Nelson, J. Lightwave Technol. 9, 598 (1990), and references therein.

4. A. Kumar and R. K. Varshney, Opt. Lett. 14, 817 (1989).

5. C. D. Poole, J. M. Wiesenfeld, A. R. McCormick, and K. T. Nelson, Opt. Lett. 17, 985 (1992).

6. R. H. Stolen and R. P. De Paula, Proc. IEEE 75, 1485 (1992).

7. A. W. Snyder and J. D. Love, Optical Waveguide Theory (Chapman \& Hall, London, 1983), Chap. 12, pp. 248-263.

8. D. Gloge, Appl. Opt. 10, 2252 (1971).

9. B. Y. Kim, J. N. Blake, S. Y. Huang, and H. J. Shaw, Opt. Lett. 12, 729 (1987).

10. S. Y. Huang, J. N. Blake, and B. Y. Kim, J. Lightwave Technol. 8, 23 (1990).

11. S. J. Garth, Appl. Opt. 28, 581 (1989).

12. F. Ouellette, IEEE J. Quantum Electron. 27, 796 (1991)

13. A. Yariv, Quantum Electronics, 2nd ed. (Wiley, New York, 1975), Chap. 3, p. 118. 